\title{
The Effects of Nitric Acid on Extraction Properties of TODGA During Fission Product Management
}

Michael A. Bromley and Colin Boxall

Engineering Department, Lancaster University, Bailrigg, Lancaster LA1 4YW, UK

\begin{abstract}
In the context of management of the wastes arising from the reprocessing of spent nuclear fuel, studies have been conducted into the solvent extraction kinetics of lanthanide fission products by TODGA and the effects of solvent phase acidity on the effectiveness of this organic extractant molecule. In this study we observe an increase in organic phase viscosity increasing acidity and, potentially by consequence, that the rate of cerium extraction is summarily decreased.
\end{abstract}

\section{INTRODUCTION}

Nuclear power remains key to energy production strategies worldwide and as such, significant research interest exists within the development of waste management strategies. Several aqueous partitioning processes have been developed to scientific feasibility in recent years (i-SANEX, EXAM, EURO-GANEX) [1] with particular aims to achieve effective recycling of minor actinides from long-lived radioactive waste, thus aiding geological disposal, while future strategies look towards the development of techniques for the dissolution, reprocessing and manufacture of mixed oxide fuels for a safe closed nuclear fuel cycle. Consequently, it is important to develop a comprehensive understanding of the organic complexants and diluents involved in such processes including their long-term suitability and potential for regeneration and recycling in order to minimize process waste and cost.

As such, we are conducting ongoing studies into the kinetics of the extraction of lanthanide fission products (as minor actinide simulants) from $\mathrm{HNO}_{3}$ by the trivalent organic extractant molecule TODGA (N,N,N',N'- tetraoctyl diglycolamide) with the use of a rotating diffusion cell (RDC) [2]. The RDC is a compartmental diffusion apparatus which enables the study of interfacial kinetics and the interrogation of several kinetic regimes from mass transport to thermodynamic control as well as the transition between the two [3].

Through use of this technique, we have quantified the interfacial kinetics of the extraction of Ce(III) by TODGA [4] and have identified that the key complexation reaction takes place in the aqueous solution phase [5]. Further observations have shown the rate of Ce extraction to have linear dependence on the aqueous concentrations of both $\mathrm{Ce}(\mathrm{III})$ and $\mathrm{HNO}_{3}$ (not shown), indicating that the extraction process occurs with the inclusion of a single nitrate ion in the first coordination sphere of the Ce(III)-TODGA complex; and, through variation of both hydrodynamic conditions and key solution concentrations, that the system operates under mixed mass transfer with chemical reaction (MTWCR) control [6]. These observations are in agreement with concurrent studies Lelias et al [4] and Simonin et al [4].

We have also observed a reduction in the extraction kinetics of the Ce(III) / TODGA system where the organic solution phase is acidified by $\mathrm{HNO}_{3}$; given the acidic environments associated with solvent extraction processes, the effects of $\left[\mathrm{HNO}_{3}\right]_{\text {org }}$ on the extraction properties of TODGA have been investigated and form the basis for this paper. 


\section{EXPERIMENTAL}

All reagents used are AnalaR grade or higher, and purchased from Sigma-Aldrich (Dorset, UK) or Alfa Aesar (Lancashire, UK) with the exceptions of TODGA (Technocomm Ltd., Falkland, UK) and Exxsol D80 (ExxonMobil Chemical Ltd, Surrey, UK). All water used is ultrapure doubly de-ionized water from a Direct-Q 3 UV Millipore water purification system (Millipore, Watford, UK) to a resistivity of $18.2 \mathrm{M} \Omega \cdot \mathrm{cm}$.

The RDC apparatus, manufactured in-house at Lancaster University, comprises two solution vessels separated by a Millipore GSWP04700 nitrocellulose membrane filter, Figure 1 (a). Membranes of $0.22 \mu \mathrm{m}$ pore size and $150 \mu \mathrm{m}$ thickness are mounted on the inner vessel using methyl-methacrylate-based adhesive and treated with clearing solvent (33\% n-hexane, $33 \%$ 1,2-dichloroethane, 33\% 1,4-dioxane, 1\% water) in order to selectively and irreversibly collapse the porous structure of the membrane, Figure 1 (b). This creates a defined area solution phase interface across which the transport of species may be studied.

Rotation of the inner RDC vessel generates hydrodynamic flow conditions at each side of the interface membrane [2]; at the outer membrane surface, these conditions closely match those of widely studied rotating disc systems without modification while a static baffle is employed in the inner vessel in order to generate a flow pattern towards and normal to the interface. Establishment of a robust and controllable hydrodynamic environment at each side of the solution phase interface is key to the study of kinetic regimes within the RDC [3].

(a)

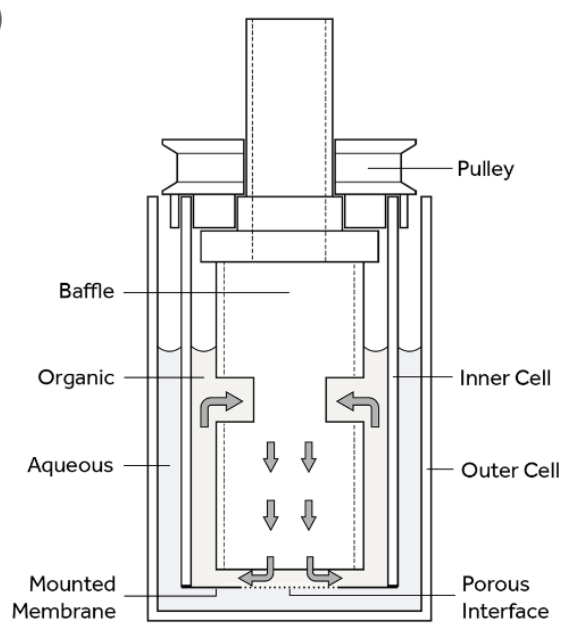

(b)

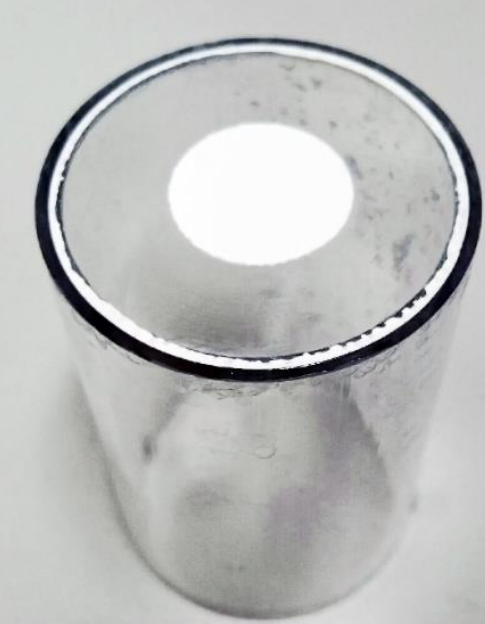

Figure 1 (a) Rotating diffusion cell schematic, (b) mounted nitrocellulose membrane interface

RDC studies are performed with a freshly prepared aqueous solution phase of 10 $\mathrm{mmol} / \mathrm{dm}^{3} \mathrm{Ce}$ (III) $\mathrm{N}_{3} \mathrm{O}_{9}$ in $1 \mathrm{~mol} / \mathrm{dm}^{3} \mathrm{HNO}_{3}$ in the outer vessel and an organic solution phase of $0.2 \mathrm{~mol} / \mathrm{dm}^{3}$ TODGA in Exxsol D80 with $5 \%$ vol 1-octanol in the inner vessel. The organic phase is allowed to fully permeate the nitrocellulose membrane, ensuring that the phase boundary is positioned at the outer membrane surface while equal solution levels are maintained to avoid any hydrostatic pressure effects across the interface. The RDC is the operated at a rotation speed of $1 \mathrm{~Hz}$ for 120 min with monitoring of each solution phase performed using a Gamry Spectro-115U UV-Vis spectrophotometer (Gamry Instruments, Cheshire, UK).

Where organic solution phases are to be used in a non-equilibrated state, no prior contact is made with the aqueous phase; where the organic phase is to be used in an $\mathrm{HNO}_{3}$ equilibrated 
or $\mathrm{H}_{2} \mathrm{O}$ washed state, contacting is performed via 5 min shaking in an Eppendorf ThermoMixer $\mathrm{C}$ (Eppendorf UK Ltd, Stevenage, UK) and 5 min centrifugation in a MicroStar 12 centrifuge (VWR, West Sussex, UK). Viscosity studies are performed using a Brookfield LV DV1 viscometer with UL adapter for low viscosity (Brookfield Viscometers Ltd, Essex, UK).

\section{DISCUSSION}

The findings of previous RDC studies in our laboratory discussed above are concerned with the kinetics of extraction of $\mathrm{Ce}$ (III) by freshly prepared, non-equilibrated TODGA, i.e. TODGA that has not been previously contacted / equilibrated with $\mathrm{HNO}_{3}$. Ongoing RDC studies with non-equilibrated TODGA again demonstrate the simultaneous extraction of both $\mathrm{Ce}$ (III) and $\mathrm{HNO}_{3}$, each detectable via $\mathrm{UV}$-Vis spectrophotometry of the organic solution phase with relative change in absorbance corresponding to a change in species concentration. $\mathrm{HNO}_{3}$ in the organic phase is represented by an increase in UV-Vis absorbance at a $\lambda_{\max }$ of $300 \mathrm{~nm}$ and shows a linear trend $v s$ time for the duration of the RDC experiments, Figure 2 (a). This demonstrates that the $\mathrm{HNO}_{3}$ extraction by TODGA occurs at a constant rate from the outset with no evidence of saturation within the experimental time frame.

Extracted Ce(III) is represented by an increase in UV-V absorbance at a $\lambda_{\max }$ of $346 \mathrm{~nm}$ which, while occurring at an initially steady rate at $t=<60 \mathrm{~min}$, begins to plateau as the rate of Ce extraction slows significantly at $\mathrm{t}=>60 \mathrm{~min}$, Figure 3 (a). This marked change is not attributable to complete extraction as depletion of the aqueous $\mathrm{Ce}$ (III) solution is not observed, nor is it attributable to having reached the loading capacity of the extractant molecule as the quantity of TODGA in the RDC system should capably extract all available Ce(III) [7].

The fact that $\mathrm{HNO}_{3}$ extraction continues at an unperturbed rate suggests that free TODGA remains available in the organic phase but the decline in $\mathrm{Ce}$ (III) extraction indicates that the capture / complexation of Ce ions becomes inhibited during the experimental process, potentially with relation to the increasing acidity of the extractant as $\mathrm{HNO}_{3}$ is transported.

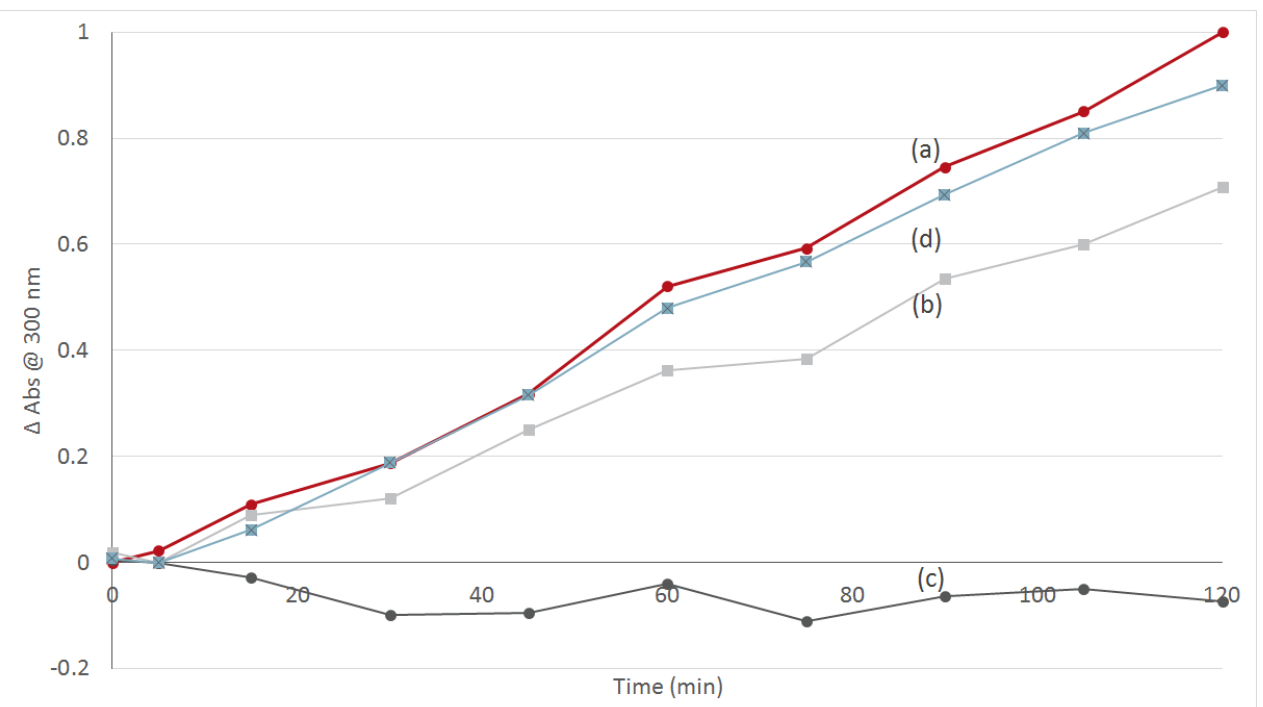

Figure 2 Plot of $\Delta\left[\mathrm{HNO}_{3}\right]_{\text {org }}$ measured as absorbance at $300 \mathrm{~nm}$ vs time for (a) non-equilibrated TODGA solution, (b) $1 \mathrm{~mol} / \mathrm{dm}^{3} \mathrm{HNO}_{3}$-equilibrated TODGA solution, (c) $5 \mathrm{~mol} / \mathrm{dm}^{3} \mathrm{HNO}_{3}$-equilibrated TODGA solution, (d) $\mathrm{H}_{2} \mathrm{O}$ washed TODGA solution. 
To investigate the effect of solvent acidity on the rate of extraction, the same RDC experiments were conducted using TODGA which was pre-contacted / pre-equilibrated with 1 $\mathrm{mol} / \mathrm{dm}^{3} \mathrm{HNO}_{3}, 5 \mathrm{~mol} / \mathrm{dm}^{3} \mathrm{HNO}_{3}$ and TODGA which was pre-contacted / pre-equilibrated and then repeatedly given a mild wash with $\mathrm{H}_{2} \mathrm{O}$ in an attempt to remove the acid. Extraction of $\mathrm{HNO}_{3}$ and $\mathrm{Ce}(\mathrm{III})$ by these three systems as functions of time are shown in Figures 2 and 3 respectively. It can be seen that acid pre-contacting has a clear influence on the subsequent extraction of both $\mathrm{HNO}_{3}$ and $\mathrm{Ce}(\mathrm{III})$ by TODGA in the RDC.

In the case of $\mathrm{HNO}_{3}$ extraction, it is observed that $1 \mathrm{~mol} / \mathrm{dm}^{3}$ equilibrated TODGA continues to extract at a linear rate albeit reduced from that of non-equilibrated TODGA, Figure 2 (B). Here, the equilibration process has pre-loaded the TODGA with $\mathrm{HNO}_{3}$ but not reached the point of saturation, hence the continued extraction of further acid in the RDC system. In contrast, the $5 \mathrm{~mol} / \mathrm{dm}^{3}$ equilibrated TODGA is completely $\mathrm{HNO}_{3}$ saturated and no further acid extraction is observed to occur during the RDC experiment. Indeed, a small amount of acid appears to be back-extracted by the $1 \mathrm{~mol} / \mathrm{dm}^{3} \mathrm{HNO}_{3}$ aqueous solution phase of the RDC, resulting in a reduction is absorbance at the $300 \mathrm{~nm}$ wavelength, Figure 2 (c). $\mathrm{H}_{2} \mathrm{O}$ washed TODGA shows $\mathrm{HNO}_{3}$ extraction which is almost comparable to that of non-equilibrated TODGA with only a small reduction in rate evident, Figure 2 (d). This suggests that although the $\mathrm{H}_{2} \mathrm{O}$ washing process does remove the vast majority of $\mathrm{HNO}_{3}$ from the organic solution phase, a residual level of acidity remains.

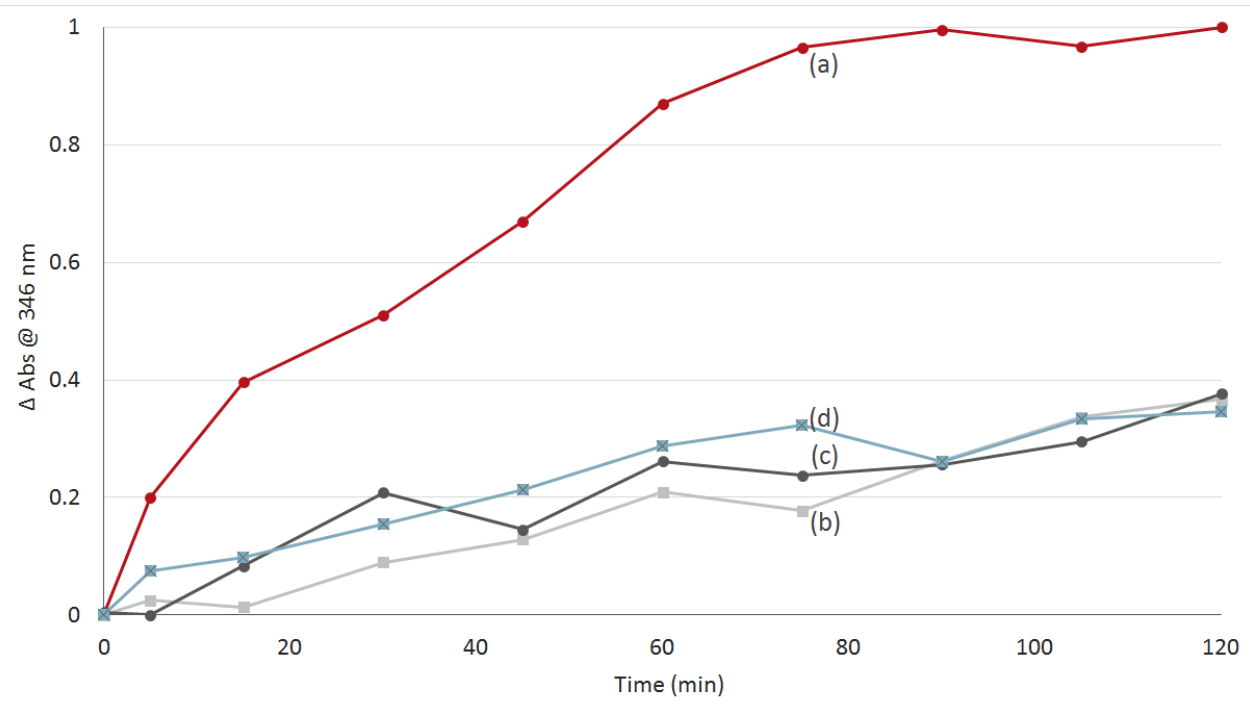

Figure 3 Plot of $\Delta[\mathrm{Ce}(\mathrm{III})]_{\text {org }}$ measured as absorbance at $346 \mathrm{~nm}$ vs time for (a) non-equilibrated TODGA solution, (b) $1 \mathrm{~mol} / \mathrm{dm}^{3} \mathrm{HNO}_{3}$-equilibrated TODGA solution, (c) $5 \mathrm{~mol} / \mathrm{dm}^{3} \mathrm{HNO}_{3}$-equilibrated TODGA solution, (d) $\mathrm{H}_{2} \mathrm{O}$ washed TODGA solution.

In the case of $\mathrm{Ce}$ (III) extraction, a different relationship is observed as the $1 \mathrm{~mol} / \mathrm{dm}^{3}$ $\mathrm{HNO}_{3}$-equilibrated, $5 \mathrm{~mol} / \mathrm{dm}^{3} \mathrm{HNO}_{3}$-equilibrated and $\mathrm{H}_{2} \mathrm{O}$ washed TODGA solutions all behave similarly to one another, Figure 3 (b,c,d), each showing a significant reduction in the rate of $\mathrm{Ce}$ (III) extraction in the RDC when compared to fresh, non-equilibrated TODGA. It is clear that in each case, the effect of pre-contacting TODGA with $\mathrm{HNO}_{3}$ results in a modification to the organic solution phase which in turn inhibits the $\mathrm{Ce}$ (III) extraction process. The lack of differentiation between the behavior of TODGA with low remaining acidity and that which is $\mathrm{HNO}_{3}$ saturated indicates that all investigated solutions are above a critical point of effect and 
that the onset of Ce extraction inhibition occurs at a very low acidity level. This maintains agreement with the earlier theory that the reduction in the rate of $\mathrm{Ce}$ (III) extraction during the RDC experiment is due to increasing acidity of the organic extractant. Given that $\mathrm{HNO}_{3}$ is extracted simultaneously from the start, it is reasonable to conclude that a critical point of acidity may be reached which then suppresses the extraction of the $\mathrm{Ce}(\mathrm{III})$.

Existing literature studies report the aggregation of TODGA into reverse micelles upon contact of the organic phase with $\mathrm{HNO}_{3}[8,9]$. These studies report the formation of tetramer reverse micelles, consisting of 4 TODGA molecules around an aqueous core, with a critical micellation point occurring with $\mathrm{HNO}_{3}$ concentrations of $\geq 0.7 \mathrm{~mol} / \mathrm{dm}^{3}$. The increase in solvent viscosity commonly observed upon micellation could have a negative effect on a diffusive mass transport dependent system such as that of the RDC; consequently, the viscosity and UV-Vis absorbance of $0.2 \mathrm{~mol} / \mathrm{dm}^{3}$ solutions of TODGA in Exxsol D80 were monitored after equilibration with increasing $\mathrm{HNO}_{3}$ concentration aqueous solutions.

The results demonstrate a clear effect with the TODGA solution showing a sigmoid increase in both absorbance at the $300 \mathrm{~nm}$ wavelength, Figure 4 (a), and organic phase viscosity, Figure 4 (b), with increasing aqueous $\mathrm{HNO}_{3}$ concentration. In the absence of TODGA, the organic phase viscosity remains unchanged, Figure 4 (d), with UV-Vis absorbance measurements also indicating a negligible amount of $\mathrm{HNO}_{3}$ entering the organic, Figure 4 (c). This demonstrates that the viscosity of the organic solution phase is increased with increasing organic acidity and that the effect is dependent of the presence of the extractant molecule. While the measured viscosity change is relatively small, increasing from a non-equilibrated viscosity of $2.960 \mathrm{cP}$ to $3.653 \mathrm{cP}$ for $5 \mathrm{~mol} / \mathrm{dm}^{3}$ equilibrated solution, this is consistent with viscosity changes during TODGA reverse micelle formation reported by Ganguly et al in which a slow viscosity increase is observed at low micellar concentrations [10]. It is also noted that the viscosity increase shows an upturn at $0.7 \mathrm{~mol} / \mathrm{dm}^{3}$, in agreement with the critical micellation point described in existing literature. As RDC experiments operate with a $1 \mathrm{~mol} / \mathrm{dm}^{3} \mathrm{HNO}_{3}$ aqueous phase, it is concluded that $\mathrm{HNO}_{3}$ extraction increases the organic acidity sufficiently to initiate the formation of TODGA reverse micelles, thus increasing viscosity and inhibiting mass-transport in the system.

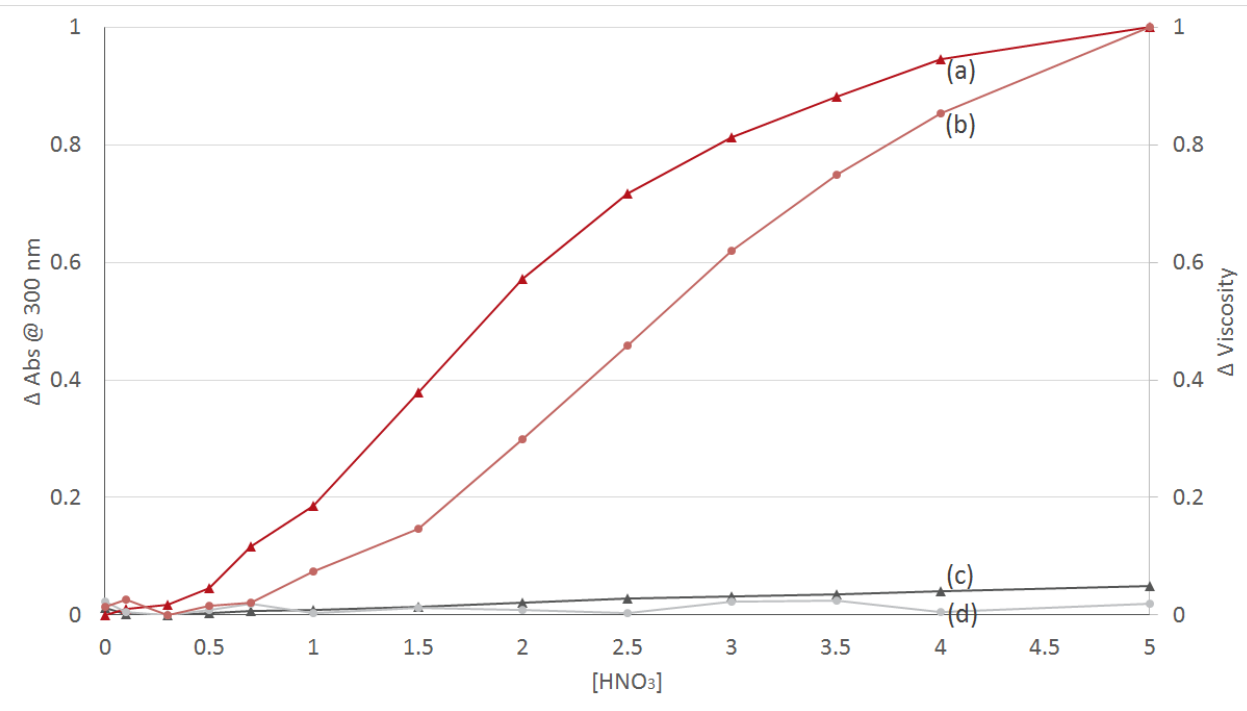

Figure 4 Plot of relative change in (a) absorbance of TODGA soln., (b) viscosity of TODGA soln., (c) absorbance of Exxsol D80 diluent and (d) viscosity of Exxsol D80 diluent. (Normalised to common axes) 
Further viscosity measurements on $\mathrm{HNO}_{3}$-equilibrated TODGA were performed after repeated $\mathrm{H}_{2} \mathrm{O}$ wash cycles. It was found that the viscosity was reduced to $3.013 \mathrm{cP}$ upon the first wash, a value comparable to that of $1 \mathrm{~mol} / \mathrm{dm}^{3} \mathrm{HNO}_{3}$-equilibrated TODGA solution (3.011 cP), with no further change observed in subsequent washes. As with the deductions made from the RDC results discussed above, it appears that a residual level of acidity remains in $\mathrm{HNO}_{3}-$ equilibrated TODGA after $\mathrm{H}_{2} \mathrm{O}$ washing and that this is sufficient to cause a change to the organic phase viscosity, attributable to the formation of reverse micelles of TODGA. These observed effects of the effectiveness of TODGA for Ce(III) extraction and the implications they may have on the use and re-use of such molecules in solvent extraction processes warrant further investigation and continue to be the subject on ongoing studies in our laboratories - particularly with respect to the use of alkaline washes to remove residual acidity from the solvent phase.

\section{CONCLUSIONS}

RDC studies of the Ce(III) extraction kinetics of TODGA have previously demonstrated the system to be under MTWCR control. The interrogation of both non-equilibrated and $\mathrm{HNO}_{3}-$ equilibrated TODGA reveals that the rate of $\mathrm{Ce}(\mathrm{III})$ extraction is significantly reduced by increased organic phase acidity while the in-process observation of this phenomenon on nonequilibrated TODGA suggests that a relatively small acidity increase is responsible. A direct relationship between increasing organic phase acidity and viscosity is also demonstrated; while the measured viscosity change is relatively small, it may be responsible for the observed reduction in the kinetic extraction rates of TODGA in the mass-transport dependent RDC system. This has clear importance for the use of such molecules in nuclear waste management processes and requires further work into the significance for mass-transport dependent techniques separations plant such as centrifugal contactors.

\section{ACKNOWLEDGMENTS}

The authors thank the European Commission 7th Framework Programme for Research \& Technological Development and the UK Engineering \& Physical Sciences Research Council (EPSRC) for providing research funded and also The Lloyd's Register Foundation (LRF) for supporting research facilities at the Lancaster University. LRF helps to protect life and property by supporting engineering-related education, public engagement and the application of research.

\section{REFERENCES}

1. G. Modolo, A. Wilden, et al., Progress in Nuclear Energy 72 (0), 107-114 (2014).

2. W. J. Albery, J. F. Burke, et al., J. Chem. Soc., Far. Trans 172 (0), 1618-1626 (1976).

3. W. J. Albery and R. A. Choudhery, J. Phys. Chem. 92 (5), 1151-1155 (1988).

4. J. P. Simonin, C. Boxall, et al., SACSESS Deliverable D12.5 - Kinetics of extr. and stripping, 2015.

5. M. A. Bromley and C. Boxall, Nukleonika 60 (4 (Pt II)), 859-864 (2015).

6. C. Boxall, Add. to SACSESS Deliverable D12.5 - Kinetics of extr. and stripping, 2015.

7. $\quad$ E. Metwally, A. S. Saleh, et al., J Radioanal Nucl Chem 286 (1), 217-221 (2010).

8. S. Nave, G. Modolo, et al., Solvent Extr. Ion Exch. 22 (4), 527-551 (2004).

9. $\quad$ M. P. Jensen, T. Yaita, et al., Langmuir 23 (9), 4765-4774 (2007).

10. R. Ganguly, J. N. Sharma, et al., J. Colloid Interface Sci. 355 (2), 458-463 (2011). 